\title{
Survival Advantage With Adjuvant Chemotherapy for Locoregionally Advanced Rectal Cancer: A Veterans Health Administration Analysis
}

\author{
Daphna Y. Spiegel, MD,b; Matthew J. Boyer, MD, PhDa; Julian C. Hong, MDa; Christina D. Williams, PhDc; \\ Michael J. Kelley, MDd,e; Joseph K. Salama, MDa; and Manisha Palta, MDa
}

\section{ABSTRACT}

\begin{abstract}
Background: Adjuvant chemotherapy (AC) after chemoradiation (CRT) and surgery for locoregionally advanced rectal cancer (LARC) is a standard of care in the United States. This study examined the role, optimal regimen, and duration of $\mathrm{AC}$ using data from the largest integrated health system in the United States. Patients and Methods: Using the Veterans Affairs Central Cancer Registry, patients with stage II-III rectal cancer diagnosed in 2001 through 2011 who received neoadjuvant CRT and surgery with or without $A C$ were identified. Kaplan-Meier analysis, log-rank tests, and propensity score (PS) adjustment analysis were used to assess survival. Results: $A$ total of 866 patients were identified; 417 received $A C$ and 449 did not (observation [OBS] group). Median follow-up was 109 months. Median disease-specific survival (DSS) was not reached. Six-year DSS was $73.7 \% ; 79.5 \%$ for the AC group versus $68.0 \%$ for the OBS group. PS-matched analysis for DSS favored AC ( $P=.0002)$. Median overall survival (OS) was 90.8 months. Six-year OS was $56.7 \%$; $64.3 \%$ for AC versus $49.6 \%$ for OBS. In PS-matched analysis, median OS was 117.4 months for $\mathrm{AC}$ and 74.3 months for OBS $(P<.0001)$. A DSS advantage was seen when comparing $\geq 4$ months with $<4$ months of $A C$ $(P=.023)$. No difference in DSS or OS was seen with single-agent versus multiagent $A C$. Conclusions: In this population of patients with LARC treated with neoadjuvant CRT and surgery, OS and DSS were improved among those treated with $A C$ versus OBS. DSS benefits were seen with $\geq 4$ months of $A C$. No additional benefit was observed with multiagent therapy. In the absence of phase III data, these findings support the use of AC for LARC.
\end{abstract}

J Natl Compr Canc Netw 2020;18(1):52-58 doi: 10.6004/jnccn.2019.7329

\footnotetext{
aDepartment of Radiation Oncology, Duke University, Durham, North Carolina; bepartment of Radiation Oncology, Beth Israel Deaconess Medical Center, Boston, Massachusetts; and ${ }^{\mathrm{C} C o o p e r a t i v e ~ S t u d i e s ~ P r o g r a m ~ E p i d e m i o l o g y ~}$ Center-Durham, Durham Veterans Administration Medical Center, ${ }^{\mathrm{d} D i v i s i o n}$ of Medical Oncology, Department of Medicine, Duke University, and 'Division of Hematology-Oncology, Medical Service, Durham Veterans Administration Medical Center, Durham, North Carolina.
}

\section{Background}

Although colon and rectal cancers are frequently grouped as a single disease entity, these malignancies have important differences in treatment approaches and patterns of recurrence. Colon cancers are managed with primary surgical resection and consideration of adjuvant chemotherapy (AC) based on patient and tumor characteristics. The role of $\mathrm{AC}$ in colon cancer is supported by the results of NSABP C01, ${ }^{1}$ a pooled analysis, ${ }^{2}$ and the MOSAIC trial ${ }^{3}$ showing improvement in disease-free survival (DFS) and overall survival (OS).

Unlike colon cancers, locoregionally advanced rectal cancer (LARC) is typically managed with neoadjuvant therapy followed by total mesorectal excision (TME). AC after chemoradiation (CRT) and TME for LARC have been incorporated into practice in the United States based on limited data. Historical data before the routine use of neoadjuvant CRT and TME reported an OS and disease-specific survival (DSS) advantage for $\mathrm{AC}$, establishing its role as the standard of care and prompting the integration of AC into future clinical trials. ${ }^{4}$

In the modern era, the role of AC for LARC is questioned because of improvements in diagnostic imaging, neoadjuvant therapy, and routine use of TME. Multiple studies, including a large meta-analysis of individual patient data derived from 4 European randomized trials, do not show a survival advantage with AC. ${ }^{5-11}$

Despite the limited data supporting the use of AC in LARC, national oncology guidelines, including those published by NCCN, advocate for a total of 6 months of perioperative systemic therapy for LARC. ${ }^{12}$ The purpose of this study was to examine the population-based outcomes of patients with LARC treated with neoadjuvant CRT followed by surgery with or without AC using data from the largest integrated US healthcare system. 


\section{Patients and Methods}

\section{Patient Selection}

Patients with histologically confirmed clinical stage II-III rectal adenocarcinoma based on contemporaneous AJCC staging were included in this analysis. Staging was based on findings from physical examination and endoscopic ultrasound, CT, or MRI. Patients diagnosed in January 2001 through April 2011 were identified in the Veterans Affairs Central Cancer Registry (VACCR). The VACCR is a mandatory nationwide database of all veterans diagnosed with cancer within the Veterans Health Administration (VHA). We linked VACCR data to VHA electronic health record data and VHA administrative databases, including the Corporate Data Warehouse (CDW) and the National Death Index (NDI). Baseline characteristics, treatment details, and vital status were obtained from the CDW. Cause of death was obtained from the NDI.

\section{Measures and Outcomes}

Patients receiving neoadjuvant concurrent CRT followed by surgery with or without AC were included in the analysis. Radiation fractionation was determined by CPT codes, limited to within 180 days of diagnosis, 1 treatment per day, and $\leq 1$ week between consecutive treatments, or by review of CDW abstracted data. Neoadjuvant radiation was defined as delivery of $>20$ total fractions. Start dates for neoadjuvant chemotherapy and radiation had to be within 30 days to ensure concurrent delivery. CRT was considered neoadjuvant if surgery occurred $<180$ days after radiation start. AC was defined as chemotherapy starting within 120 days of surgery. NCCN Clinical Practice Guidelines in Oncology (NCCN Guidelines) for Rectal Cancer recommend a total of 6 months of perioperative systemic therapy, which includes a course of conventionally fractionated CRT and 4 additional months of $\mathrm{AC}$, as one approach to the treatment of LARC. ${ }^{12}$ Therefore, analyses evaluating duration of AC were defined by a cut point of $\geq 4$ months of therapy. The study was approved by Durham Veteran's Affairs Institutional Review Board.

\section{Statistical Analysis}

Differences in baseline characteristics were determined using the Pearson chi-square test or $t$ test for categorical or continuous variables, respectively. The primary endpoints were DSS and OS, calculated from the diagnosis date to the date of death. Death caused by disease was defined by ICD-10 codes C18.9, C19, and C20. To account for possible immortal time bias due to the interval between surgery and start of AC, a landmark analysis was also performed for DSS and OS. ${ }^{13,14}$ A 6.3-month landmark from the date of surgery was selected because this included the time interval whereby $90 \%$ of the patients who received AC initiated this treatment. The landmark analysis included only patients who were alive and followed at 6.3 months after surgery. ${ }^{15}$ DSS and OS were estimated using the Kaplan-Meier method, and comparisons were performed using the log-rank test.

Propensity score (PS) adjustment was used to compare survival outcomes adjusted for differences in baseline characteristics. The PS was calculated based on a multiple logistic regression for receiving a given treatment. The model included the following variables: age, race, sex, smoking status, Charlson-Deyo comorbidity index (CCI) score, and AJCC stage. CCI score was calculated using ICD-9 codes in the year before diagnosis. ${ }^{16,17}$ Matched pair analysis was performed using the logit of the estimated PS. STATA/MP versions 14.2 and 15.0 (StataCorp LLP) and SAS 9.4 (SAS Institute Inc) were used for analysis.

\section{Results}

\section{Patient Characteristics}

Between January 1, 2001, and April 30, 2011, we identified 58,593 patients in the VACCR who were diagnosed with colorectal cancer; 2,784 were identified as having stage II-III rectal adenocarcinoma and were treated with CRT, and 1,000 subsequently underwent surgery either with or without AC. Of this final sample, 417 received AC, 449 received no further therapy and were included in the observation (OBS) group, and AC data were not available for 134 patients (Figure 1). Median patient age was 62.4 years (interquartile range [IQR], 12.5 ), $84.4 \%$ were white, $98.7 \%$ were male, and $47.8 \%$ had stage III disease. Patients treated with AC were more likely to have higher AJCC stage at diagnosis $(P=.014)$; however, no differences were seen in baseline age, race, sex, smoking status, or CCI score (Table 1).

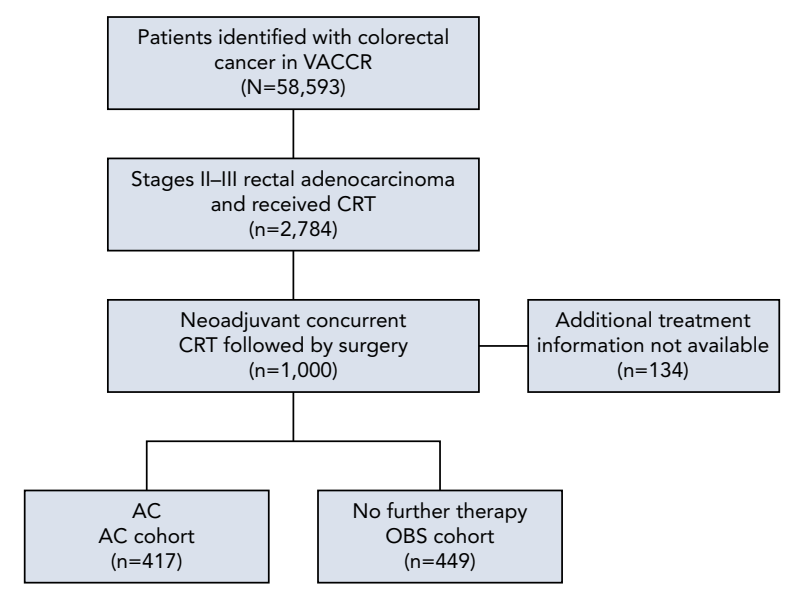

Figure 1. Cohort assembly diagram.

Abbreviations: AC, adjuvant chemotherapy; CRT, chemoradiation; OBS, observation; VACCR, Veterans Affairs Central Cancer Registry. 


\begin{tabular}{|c|c|c|c|}
\hline Characteristic & $\begin{array}{c}\mathrm{AC} \\
\mathrm{n}(\%)\end{array}$ & $\begin{array}{c}\text { No AC } \\
\text { n (\%) }\end{array}$ & $\begin{array}{c}P \\
\text { Value }\end{array}$ \\
\hline Patients, N & 417 & 449 & \\
\hline \multicolumn{4}{|l|}{ Age, y } \\
\hline Median & 61.5 & 63.0 & \\
\hline IQR & 11.1 & 13.8 & \\
\hline Race & & & .279 \\
\hline White & $343(82.2)$ & $388(86.4)$ & \\
\hline Black & $62(14.9)$ & $48(10.7)$ & \\
\hline Other & $12(2.8)$ & $15(3.3)$ & \\
\hline Sex & & & .431 \\
\hline Male & $413(99.0)$ & $442(98.4)$ & \\
\hline Female & $4(1.0)$ & $7(1.6)$ & \\
\hline Smoking status & & & .578 \\
\hline Yes (current or former) & 307 (73.6) & $323(71.9)$ & \\
\hline Never & $110(26.4)$ & $126(28.1)$ & \\
\hline AJCC stage & & & .014 \\
\hline$\| \mathrm{A}$ & $173(41.5)$ & $232(51.7)$ & \\
\hline$\| B$ & 19 94.6) & $28(6.2)$ & \\
\hline III & $11(2.6)$ & $13(2.9)$ & \\
\hline IIIA & $28(6.7)$ & $15(3.3)$ & \\
\hline IIIB & 135 (32.4) & $123(27.4)$ & \\
\hline IIIC & $51(12.2)$ & $38(8.5)$ & \\
\hline $\mathrm{CCl}$ score & & & .384 \\
\hline 0 & $215(51.6)$ & $207(46.1)$ & \\
\hline $1-2$ & $155(37.2)$ & $167(37.2)$ & \\
\hline $3-4$ & $40(9.6)$ & $58(12.9)$ & \\
\hline$\geq 5$ & $7(1.7)$ & $17(3.8)$ & \\
\hline
\end{tabular}

Abbreviations: $\mathrm{AC}$, adjuvant chemotherapy; $\mathrm{CCl}$, Charlson-Deyo comorbidity index; IQR, interquartile range.

aBold indicates a statistically significant result.

Median duration of AC was 3.63 months (IQR, 2.7) (Figure 2). Of the patients who received AC, $42.2 \%$ were treated with multiagent chemotherapy: $60.8 \%$ received 5-FU/oxaliplatin (FOLFOX), 26.7\% received capecitabine/oxaliplatin (CAPEOX), and $12.5 \%$ received a different multiagent regimen (FOLFIRI [leucovorin/5-FU/irinotecan], FOLFIRINOX [leucovorin/ 5-FU/irinotecan/oxaliplatin], FOLFIRINOX + bevacizumab, FOLFOX + bevacizumab, or CAPEOX + bevacizumab). Of those who received single-agent chemotherapy, $63.9 \%$ were treated with 5-FU and the remainder were treated with capecitabine. Patients treated with multiagent chemotherapy were more likely to have higher AJCC stage at diagnosis $(P<.0001)$, but no differences were seen in baseline age, race, sex, smoking status, or CCI score between the treatment cohorts (Table 2).

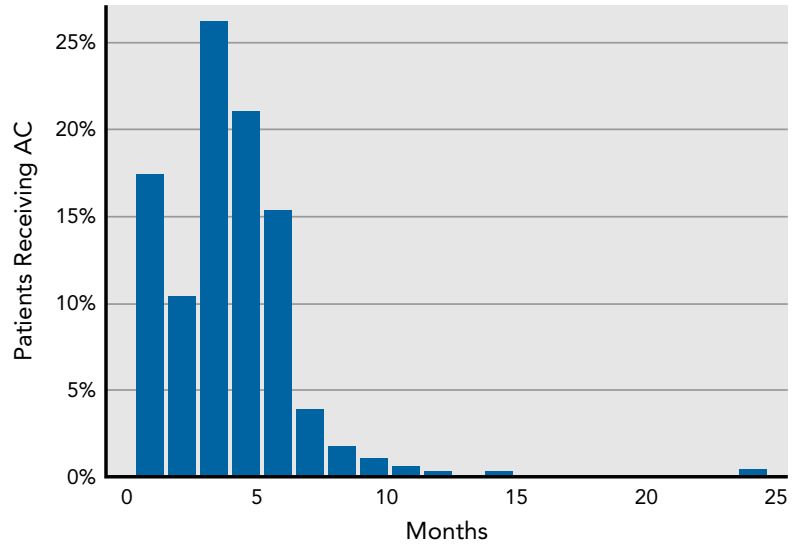

Figure 2. Duration of adjuvant chemotherapy (AC).

\section{Survival Outcomes}

Median follow-up for all patients was 109 months. Median DSS was not reached for all patients. Six-year DSS for all patients was 73.7\% (95\% CI, 70.5-76.7). DSS at 6 years was $79.5 \%$ (95\% CI, 75.1-83.2) for the AC group versus $68.0 \%$ (95\% CI, 63.2-72.4) for the OBS group. AC improved DSS compared with OBS in unmatched analysis (hazard ratio [HR], 0.61; 95\% CI, 0.46-0.79; $P=.0002)$. In PS-matched analysis, an improvement in DSS was seen with AC compared with OBS (HR, 0.59; 95\% CI, 0.44-0.77; $P=.0002$ ) (Figure 3A). The DSS advantage for AC persisted in landmark analysis, with median DSS not reached for both the AC and OBS groups (HR, 0.59; 95\% CI, 0.44-0.78; $P=.0002$ ).

For all patients, median OS was 90.8 months $(95 \% \mathrm{CI}$, 80.1-100.1) and 6-year OS was 56.7\% (95\% CI, 53.3-59.9). OS at 6 years was $64.3 \%$ (95\% CI, 59.9-68.7) for AC and $49.6 \%$ (95\% CI, 44.9-54.1) for OBS. Unmatched analysis showed that AC improved OS compared with OBS (HR, 0.66; 95\% CI, 0.55-0.79; $P<.0001)$. In PS-matched analysis, median OS was 117.4 months (95\% CI, 98.8-145.0) for the AC group and 74.3 months (95\% CI, 66.3-84.8) for the OBS group (HR, 0.67; 95\% CI, 0.56-0.81; $P<.0001$ ) (Figure 3B). The survival advantage for AC persisted in landmark analysis, with a median OS of 111.0 months (95\% CI, 93.7-138.7) for the AC group and 70.6 months (95\% CI, 61.6-82.1) for the OBS group (HR, 0.69; 95\% CI, $0.57-0.83 ; P=.0001)$.

A total of 76 patients $(8.8 \%)$ had a pathologic complete response (ypCR, including both ypTis and ypT0) after neoadjuvant CRT. No OS or DSS benefit was seen for AC in patients with ypCR (OS: HR, 0.86; 95\% CI, 0.41-1.79; $P=.680$; and DSS: HR, $0.44 ; 95 \%$ CI, $0.12-1.72$; $P=.240$ ). Patients who did not achieve a ypCR to neoadjuvant therapy did experience an OS and a DSS benefit with the addition of AC (OS: HR, 0.65; 95\% CI, 0.54-0.78; $P<.0001$; and DSS: HR, 0.61; 95\% CI, 0.46-0.80; $P<.0001$ ). 


\begin{tabular}{|c|c|c|c|}
\hline Characteristic & $\begin{array}{c}\text { Single-Agent } \\
\text { Chemotherapy } \\
\text { n (\%) }\end{array}$ & $\begin{array}{c}\text { Multiagent } \\
\text { Chemotherapy } \\
\text { n (\%) }\end{array}$ & $P$ Value $^{a}$ \\
\hline Patients, N & $241(57.8)$ & $176(42.2)$ & \\
\hline \multicolumn{4}{|l|}{ Age, y } \\
\hline Median & 61.6 & 60.3 & \\
\hline IQR & 10.9 & 8.7 & \\
\hline Race & & & .609 \\
\hline White & $202(83.8)$ & $141(81.1)$ & \\
\hline Black & $32(13.2)$ & $30(17.0)$ & \\
\hline Other & $7(2.9)$ & $5(2.9)$ & \\
\hline Sex & & & .484 \\
\hline Male & $238(98.8)$ & $175(99.4)$ & \\
\hline Female & $3(1.2)$ & $1(0.6)$ & \\
\hline Smoking status & & & .421 \\
\hline Yes (current or former) & $181(75.1)$ & $126(71.6)$ & \\
\hline Never & $60(24.9)$ & $50(28.4)$ & \\
\hline AJCC stage & & & $<.0001$ \\
\hline$\| A$ & $113(46.9)$ & $60(34.1)$ & \\
\hline IIB & $11(4.6)$ & $8(4.5)$ & \\
\hline III & $9(3.7)$ & $2(1.1)$ & \\
\hline IIIA & $15(6.2)$ & $13(7.4)$ & \\
\hline IIIB & $79(32.8)$ & $56(31.8)$ & \\
\hline IIIC & $14(5.8)$ & $37(21.0)$ & \\
\hline $\mathrm{CCl}$ score & & & .618 \\
\hline 0 & $127(52.7)$ & $88(50.0)$ & \\
\hline $1-2$ & $88(36.5)$ & $67(38.1)$ & \\
\hline $3-4$ & $24(10.0)$ & $16(9.1)$ & \\
\hline$\geq 5$ & $2(0.8)$ & $5(2.8)$ & \\
\hline
\end{tabular}

Abbreviations: $\mathrm{CCl}$, Charlson-Deyo comorbidity index; IQR, interquartile range.

aBold indicates a statistically significant result.

\section{Duration and Choice of Systemic Therapy}

Additional analyses were performed to evaluate differences in DSS and OS relative to systemic therapy duration and regimen. A significant DSS advantage was seen when comparing $\geq 4$ versus $<4$ months of $\mathrm{AC}$ (HR, 0.61; 95\% CI, 0.39-0.93; $P=.023$ ) (Figure 4A), but no OS advantage (HR, 0.81; 95\% CI, 0.61-1.08; $P=.145$ ) (Figure 4B). The benefit of $\geq 4$ months of AC persisted in landmark DSS analysis (HR, $0.63 ; 95 \% \mathrm{CI}, 0.41-0.97 ; P=.037$ ). No significant difference in DSS (HR, 1.15; 95\% CI, 0.75-1.75; $P=.534$ ) or OS (HR, 1.22; $95 \%$ CI, $0.91-1.65 ; P=.187$ ) was seen when comparing singleagent versus multiagent chemotherapy (Figure 5). When stratified by disease stage, no differences in DSS or OS were seen with single-agent versus multiagent chemotherapy (DSS for stage II: HR, 1.12; 95\% CI, $0.53-2.35 ; P=.772$;
DSS for stage III: HR, 1.22; 95\% CI, 0.72-2.06; $P=.458$; OS for stage II: HR, 0.92; 95\% CI, 0.57-1.49; $P=.746$; OS for stage III: HR, 1.16; 95\% CI, 0.80-1.68; $P=.433$ ).

\section{Discussion}

Findings of our study showed that the use of AC was associated with improvement in both OS and DSS in a PS-matched analysis. AC was most beneficial in patients who did not achieve ypCR. Furthermore, we found that at least 4 months of systemic therapy was needed to achieve a DSS improvement and that there was no additional benefit to multiagent over single-agent chemotherapy. To our knowledge, this is the first study to show a survival advantage for AC compared with OBS after neoadjuvant CRT and surgery for LARC.

Strengths of this analysis include high-quality data contained in the mandatorily collected VHA database and the large patient numbers. Although nonrandom assignment of AC versus OBS could have introduced sources of biases into our study, we used multiple approaches to mitigate this. A PS analysis was performed, which is a method designed to help reduce bias by accounting for the covariates that predict receiving the treatment that is being studied. We do acknowledge, however, that there are limitations to PS matching, including selection of variables to include in the PS. Certain factors that could have impacted outcomes, including poor performance status after neoadjuvant CRT, postsurgical complications, and response to neoadjuvant treatment, could have influenced adjuvant treatment decisions and, due to the retrospective nature of these data, were unable to be accounted for in the PS. We also performed a landmark analysis to correct for possible immortal time bias because of the interval between surgery and start of AC. Another potential source of bias includes the small number of women and minorities represented in the VHA database, although prior studies have shown that outcomes of VHA patients with cancer are generalizable to fee-for-service Medicare beneficiaries. ${ }^{18}$ In addition, the type of surgery-TME versus non-TME resection-was not routinely coded in the VACCR, which may have impacted clinical outcomes, although all patients had either an abdominoperineal resection or low anterior resection. Finally, other biases could be related to stage migration and changes in treatment paradigms over time, particularly given that the database includes patients diagnosed before 2004, when the results of the MOSAIC trial were published. ${ }^{2,3}$ Despite our efforts to account for correctable biases, there are potential unknown confounders, inherent to any retrospective study, that could have impacted the findings.

Our data differ from those from a number of prior analyses. Although randomized trials have evaluated the role of AC in LARC, none have shown a DSS or OS benefit for AC..$^{5,6,8-11}$ The EORTC 22921 study randomly assigned patients with LARC to preoperative radiotherapy alone, 
A

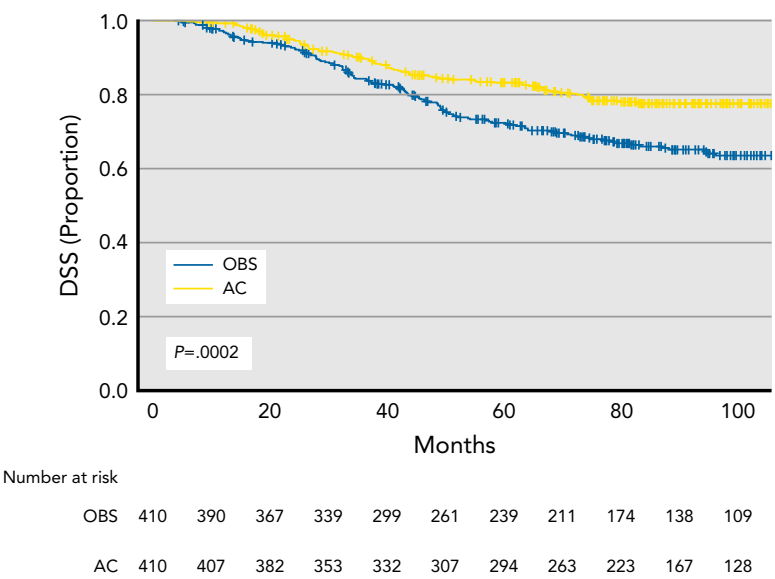

B

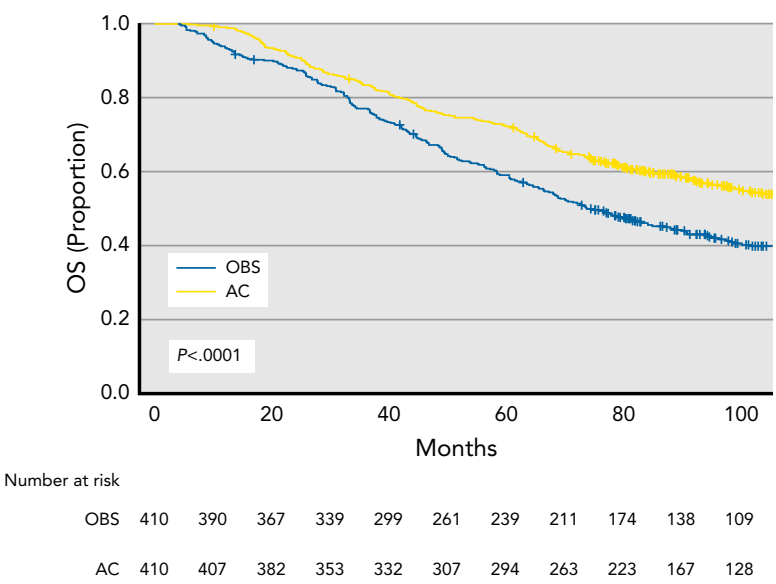

Figure 3. Matched (A) DSS and (B) OS for AC versus OBS.

Abbreviations: AC, adjuvant chemotherapy; DSS, disease-specific survival; OBS, observation (no AC); OS, overall survival.

preoperative CRT alone, preoperative RT plus postoperative chemotherapy, or preoperative CRT plus postoperative chemotherapy. ${ }^{5,6}$ These data established that the addition of chemotherapy in both the neoadjuvant and adjuvant settings decreases local recurrence, but despite long-term follow-up, there was no effect on OS or DFS with the addition of AC compared with no AC ( $P=.32$ and $P=.29$, respectively). Notably, this study did not directly compare AC with OBS in patients treated with neoadjuvant CRT. In addition, the trial design has been criticized for being underpowered and for using a bolus 5-FU regimen that is not standard in the United States.,5 In a randomized trial performed by Cionini et al, ${ }^{10}$ patients treated with neoadjuvant CRT followed by surgery were randomly assigned to 6 cycles of 5 -FU versus OBS. At 5 years of follow-up, no difference was seen in OS, local recurrence, or distant metastases.
This trial also used a bolus 5-FU regimen. The PROCTORSCRIPT trial randomly assigned patients with stage II or III rectal cancer who received neoadjuvant radiotherapy or CRT and TME to either AC or OBS. The trial accrued 437 eligible patients but closed early due to poor accrual. After a median follow-up of 5 years, no difference was seen in OS, DFS, local recurrence, or distant metastasis. ${ }^{9}$ An additional study from the United Kingdom randomly assigned patients to adjuvant capecitabine and oxaliplatin for 6 cycles versus OBS after neoadjuvant CRT and surgery. ${ }^{11}$ Similar to the PROCTOR-SCRIPT trial, this study closed prematurely after accruing 113 of an intended 800 patients. No differences were observed in OS or DFS.

The prevalence of LARC and lack of robust data supporting AC create a clinical conundrum for oncologists. In an effort to improve statistical power through patient numbers, a meta-analysis of individual patient
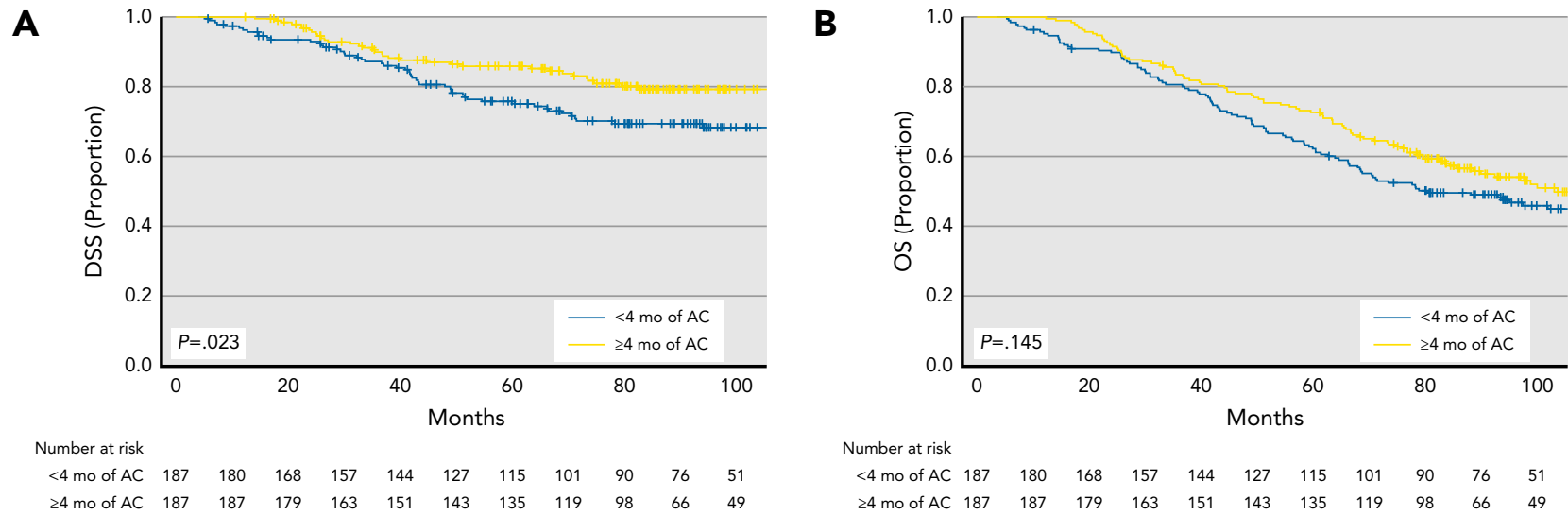

Figure 4. Matched (A) DSS and (B) OS for $\geq 4$ versus $<4$ months of $A C$

Abbreviations: AC, adjuvant chemotherapy; DSS, disease-specific survival; OS, overall survival. 
A

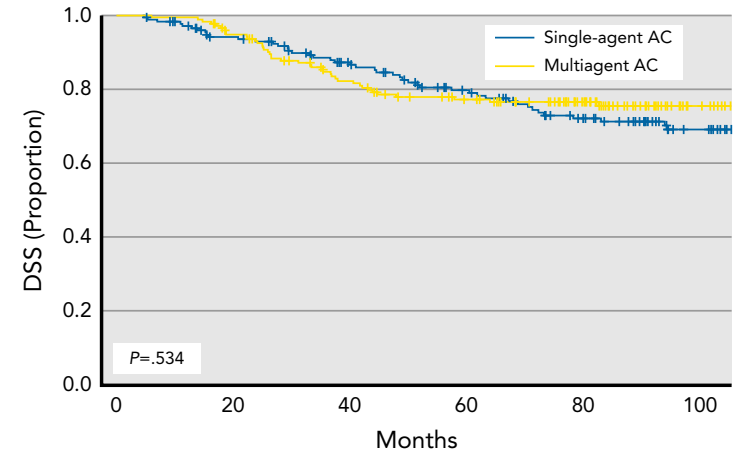

Number at risk

$\begin{array}{llllllllllll}\text { Single-agent AC } & 175 & 169 & 155 & 144 & 131 & 120 & 109 & 99 & 86 & 75 & 60\end{array}$

$\begin{array}{llllllllllll}\text { Multiagent AC } & 175 & 174 & 162 & 146 & 134 & 122 & 116 & 106 & 83 & 50 & 31\end{array}$
B

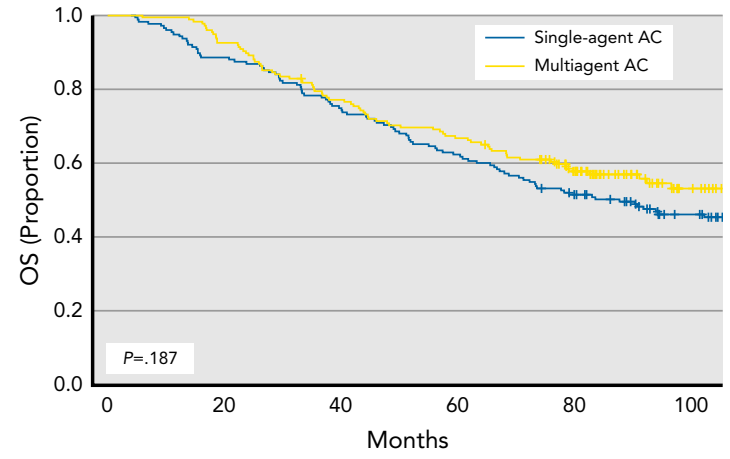

Number at risk

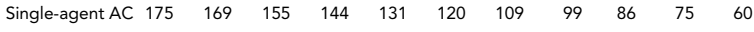

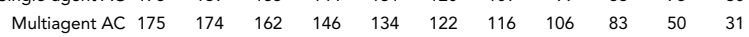

Figure 5. Matched (A) DSS and (B) OS for single-agent versus multiagent chemotherapy.

Abbreviations: AC, adjuvant chemotherapy; DSS, disease-specific survival; OS, overall survival.

data from 4 European randomized trials attempted to clarify the role of AC. This analysis included 1,196 patients with stage II or III rectal cancer receiving neoadjuvant therapy and surgical resection. No differences were seen in OS, DFS, or distant metastasis. ${ }^{8}$ Collectively, these data do not support the routine use of AC in patients with LARC treated with neoadjuvant CRT and surgery. Notably, one recently published meta-analysis did show improved OS and DFS with the addition of AC, although the benefit was derived mainly from retrospective evidence. ${ }^{19}$ Despite the limited data, US guidelines still include AC as a potential treatment option. ${ }^{12}$

The data presented in this study provide a rational justification for the use of AC and show specific patient groups that benefit. In this analysis, patients who achieved a ypCR to neoadjuvant therapy did not derive a survival advantage from AC; however, those who did not achieve a ypCR had improvements in both OS and DSS with AC. These results differ from those of the EORTC subset analysis 5,6 and 2 recently published PS-matched analyses comparing AC with OBS in patients with a ypCR after neoadjuvant therapy, ${ }^{20,21}$ suggesting that in our study, patients with persistent disease despite up-front therapy require additional systemic therapy, or that the metastatic potential of CRT responders is different from that of nonresponders.

In addition, we found that a minimum 4-month duration of AC was associated with improved DSS. This is important because compliance with AC is challenging and ranges from $43 \%$ to $73 \%$ in previous series of multiagent AC. $6,8,9,11,22$ Although other factors may also have played a role, the limited compliance with chemotherapy regimens likely contributed to the negative results of prior studies examining the benefit of AC. Furthermore, given that treatment-related adverse effects are more common with multiagent regimens, our data suggest that single-agent AC in LARC may represent the appropriate balance of efficacy and toxicity. This must be taken in a broader context, however, given that the ADORE trial did show a DFS benefit to multiagent FOLFOX chemotherapy over 5-FU alone in patients with pathologic stage III disease after neoadjuvant CRT and TME. ${ }^{23}$

In an effort to increase compliance and minimize toxicity for patients with LARC, multiple alternative treatment regimens have been considered. The PROSPECT trial, which is currently accruing patients, is examining the role of radiotherapy by randomizing patients to chemotherapy alone followed by TME versus CRT and TME (ClinicalTrials.gov identifier: NCT01515787). In Europe, short-course radiotherapy together with timing of chemotherapy are being studied in the RAPIDO trial (ClinicalTrials.gov identifier: NCT01558921), which is randomizing patients to standard long-course CRT followed by TME with AC at physicians' discretion versus short-course radiation followed by 6 cycles of multiagent chemotherapy and TME. ${ }^{24}$

Another solution to address compliance issues with AC would be to modify therapy sequence with longcourse CRT. The concept of induction chemotherapy is not new in the setting of LARC. Multiple European studies have investigated the use of induction chemotherapy, with compliance ranging from $84 \%$ to $97 \%$, which is far better than in the adjuvant setting. Furthermore, completion of neoadjuvant CRT or probability of R0 resection was not impacted. ${ }^{25-28}$ An ongoing phase II trial from Memorial Sloan Kettering Cancer Center (ClinicalTrials.gov identifier: NCT02008656) is examining the approach of total neoadjuvant therapy, ${ }^{29}$ which may allow patients to receive the total course of chemotherapy necessary to improve survival while minimizing compliance issues.

\section{Conclusions}

Our PS-matched analysis showed that the addition of AC improved both OS and DSS compared with OBS in a PSmatched analysis. In further analysis, DSS benefits were 
seen only for patients who had completed $\geq 4$ months of AC. Although AC treatment decisions should be made based on patient and tumor characteristics, these data affirm current clinical practice and support ongoing studies examining a total neoadjuvant therapy approach.

\section{Acknowledgments}

The authors wish to thank Fatima Rangwala, MD; Brian Czito, MD; and Christopher Willett, MD.

Submitted March 4, 2019; accepted for publication June 11, 2019
Previous presentation: Data was previously presented at the 2018 ASCO Gastrointestinal Cancers Symposium; January 18-20, 2018; San Francisco, California.

Author contributions: Study concept: Spiegel, Kelley, Salama, Palta. Data curation: Spiegel, Boyer, Hong, Williams. Formal analysis: Spiegel, Boyer, Hong, Salama, Palta. Investigation, validation, and visualization: Spiegel. Methodology: All authors. Supervision: Kelley, Salama, Palta. Project administration: Spiegel, Kelley, Salama, Palta. Resources and software: Spiegel, Kelley, Salama, Palta. Writing of original manuscript: Spiegel. Manuscript review and editing: All authors.

Disclosures: The authors have not received any financial consideration from any person or organization to support the preparation, analysis, results, or discussion of this article.

Correspondence: Daphna Y. Spiegel, MD, Department of Radiation Oncology, Beth Israel Deaconess Medical Center, 330 Brookline Avenue, Boston, MA 02215. Email: dspiege1@bidmc.harvard.edu

\section{References}

1. Wolmark N, Fisher B, Rockette H, et al. Postoperative adjuvant chemotherapy or BCG for colon cancer: results from NSABP protocol C-01. J Natl Cancer Inst 1988;80:30-36.

2. André $T$, Boni $C$, Mounedji-Boudiaf $L$, et al. Oxaliplatin, fluorouracil, and leucovorin as adjuvant treatment for colon cancer. N Engl J Med 2004; 350:2343-2351.

3. André $T$, Boni $C$, Navarro $M$, et al. Improved overall survival with oxaliplatin, fluorouracil, and leucovorin as adjuvant treatment in stage II or III colon cancer in the MOSAIC trial. J Clin Oncol 2009;27:3109-3116.

4. Fisher B, Wolmark N, Rockette $\mathrm{H}$, et al. Postoperative adjuvant chemotherapy or radiation therapy for rectal cancer: results from NSABP protocol R-01. J Natl Cancer Inst 1988;80:21-29.

5. Bosset JF, Calais G, Mineur L, et al. Enhanced tumorocidal effect of chemotherapy with preoperative radiotherapy for rectal cancer: preliminary results-EORTC 22921. J Clin Oncol 2005;23:5620-5627.

6. Bosset JF, Calais G, Mineur L, et al. Fluorouracil-based adjuvant chemotherapy after preoperative chemoradiotherapy in rectal cancer: long-term results of the EORTC 22921 randomised study. Lancet Oncol 2014;15:184-190.

7. Bosset JF, Collette L, Calais G, et al. Chemotherapy with preoperative radiotherapy in rectal cancer. N Engl J Med 2006;355:1114-1123.

8. Breugom AJ, Swets M, Bosset JF, et al. Adjuvant chemotherapy after preoperative (chemo)radiotherapy and surgery for patients with rectal cancer: a systematic review and meta-analysis of individual patient data. Lancet Oncol 2015;16:200-207.

9. Breugom AJ, van Gijn W, Muller EW, et al. Adjuvant chemotherapy for rectal cancer patients treated with preoperative (chemo)radiotherapy and total mesorectal excision: a Dutch Colorectal Cancer Group (DCCG) randomized phase III trial. Ann Oncol 2015;26:696-701.

10. Cionini L, Sainato A, De Paoli A, et al. Final results of randomized trial on adjuvant chemotherapy after preoperative chemoradiation in rectal cancer [abstract]. Radiother Oncol 2010;96(Suppl):S113-114.

11. Glynne-Jones R, Counsell N, Quirke P, et al. Chronicle: results of a randomised phase III trial in locally advanced rectal cancer after neoadjuvant chemoradiation randomising postoperative adjuvant capecitabine plus oxaliplatin (XELOX) versus control. Ann Oncol 2014;25:1356-1362.

12. Benson AB III, Venook AP, Al-Hawary MM, et al. NCCN Clinical Practice Guidelines in Oncology: Rectal Cancer. Version 1.2019. Accessed April 19, 2019. To view the most recent version, visit NCCN.org.

13. Dafni U. Landmark analysis at the 25 -year landmark point. Circ Cardiovasc Qual Outcomes 2011;4:363-371.

14. Giobbie-Hurder A, Gelber RD, Regan MM. Challenges of guarantee-time bias. J Clin Oncol 2013;31:2963-2969.

15. de Azambuja E, Holmes AP, Piccart-Gebhart M, et al. Lapatinib with trastuzumab for HER2-positive early breast cancer (NeoALTTO): survival outcomes of a randomised, open-label, multicentre, phase 3 trial and their association with pathological complete response. Lancet Oncol 2014;15:1137-1146.

16. Charlson ME, Pompei $P$, Ales $K L$, et al. A new method of classifying prognostic comorbidity in longitudinal studies: development and validation. J Chronic Dis 1987;40:373-383.
17. Marventano S, Grosso G, Mistretta A, et al. Evaluation of four comorbidity indices and Charlson comorbidity index adjustment for colorectal cancer patients. Int J Colorectal Dis 2014;29:1159-1169.

18. Landrum MB, Keating NL, Lamont EB, et al. Survival of older patients with cancer in the Veterans Health Administration versus fee-for-service Medicare. J Clin Oncol 2012:30:1072-1079.

19. Petrelli F, Coinu A, Lonati $V$, et al. A systematic review and meta-analysis of adjuvant chemotherapy after neoadjuvant treatment and surgery for rectal cancer. Int J Colorectal Dis 2015;30:447-457.

20. Polanco PM, Mokdad AA, Zhu H, et al. Association of adjuvant chemotherapy with overall survival in patients with rectal cancer and pathologic complete response following neoadjuvant chemotherapy and resection. JAMA Oncol 2018;4:938-943.

21. Dossa F, Acuna SA, Rickles AS, et al. Association between adjuvant chemotherapy and overall survival in patients with rectal cancer and pathological complete response after neoadjuvant chemotherapy and resection. JAMA Oncol 2018;4:930-937.

22. Sainato A, Cernusco Luna Nunzia $V$, Valentini $V$, et al. No benefit of adjuvant fluorouracil leucovorin chemotherapy after neoadjuvant chemoradiotherapy in locally advanced cancer of the rectum (LARC): long term results of a randomized trial (I-CNR-RT). Radiother Oncol 2014;113 223-229.

23. Hong YS, Nam BH, Kim KP, et al. Oxaliplatin, fluorouracil, and leucovorin versus fluorouracil and leucovorin as adjuvant chemotherapy for locally advanced rectal cancer after preoperative chemoradiotherapy (ADORE): an open-label, multicentre, phase 2, randomised controlled trial. Lancet Oncol 2014;15:1245-1253.

24. Nilsson PJ, van Etten B, Hospers GA, et al. Short-course radiotherapy followed by neo-adjuvant chemotherapy in locally advanced rectal cancer-the RAPIDO trial. BMC Cancer 2013;13:279.

25. Chau I, Brown G, Cunningham D, et al. Neoadjuvant capecitabine and oxaliplatin followed by synchronous chemoradiation and total mesorectal excision in magnetic resonance imaging-defined poor-risk rectal cancer. J Clin Oncol 2006;24:668-674.

26. Fernández-Martos C, Pericay C, Aparicio J, et al. Phase II, randomized study of concomitant chemoradiotherapy followed by surgery and adjuvant capecitabine plus oxaliplatin (CAPOX) compared with induction CAPOX followed by concomitant chemoradiotherapy and surgery in magnetic resonance imaging-defined, locally advanced rectal cancer: Grupo Cáncer de Recto 3 study. J Clin Oncol 2010;28: 859-865.

27. Calvo FA, Serrano FJ, Diaz-González JA, et al. Improved incidence of pTO downstaged surgical specimens in locally advanced rectal cancer (LARC) treated with induction oxaliplatin plus 5-fluorouracil and preoperative chemoradiation. Ann Oncol 2006;17:1103-1110.

28. Hajj C, Cercek A, Saltz L, et al. Induction chemotherapy followed by chemoradiotherapy and total mesenteric excision for locally advanced rectal cancer with resectable metastases [abstract]. J Clin Oncol 2013;31(4 Suppl):Abstract 526.

29. George TJ, Yothers G, Hong TS, et al. NRG-GI002: a phase II clinical trial platform for total neoadjuvant therapy (TNT) in rectal cancer [abstract]. J Clin Oncol 2017;35(15 Suppl):Abstract TPS3629. 